\title{
Théorie spectrale et limite semi-classique sur des variétés à pointes
}

Yannick Guedes Bonthonneau

\section{(2) OpenEdition}

Édition électronique

URL : https://journals.openedition.org/annuaire-cdf/16557

DOI : $10.4000 /$ annuaire-cdf. 16557

ISBN : 978-2-7226-0572-5

ISSN : 2109-9227

Éditeur

Collège de France

Édition imprimée

Date de publication : 30 décembre 2020

Pagination : 689-690

ISBN : 978-2-7226-0516-9

ISSN : 0069-5580

\section{Référence électronique}

Yannick Guedes Bonthonneau, «Théorie spectrale et limite semi-classique sur des variétés à

pointes », L'annuaire du Collège de France [En ligne], 118 | 2020, mis en ligne le 01 avril 2021, consulté le 22 août 2022. URL : http://journals.openedition.org/annuaire-cdf/16557 ; DOI : https://doi.org/

10.4000/annuaire-cdf. 16557 


\title{
THÉORIE SPECTRALE ET LIMITE SEMI-CLASSIQUE SUR DES VARIÉTÉS À POINTES
}

\author{
Yannick GuedES BONTHONNEAU \\ Chargé de recherche au CNRS
}

Mots-clés : théorie spectrale, limite semi-classique, variétés à pointes

Conférences prononcées les 5, 12, 19 et 26 mars 2018 (prix et cours Claude-Antoine Peccot) et disponibles en vidéo sur le site internet du Collège de France : https:// www.college-de-france.fr/site/cours-peccot/guestlecturer-2018-03-05-10h00.htm.

L'objectif de ces quatre conférences était de présenter quelques résultats en les replaçant dans leur contexte, et surtout en expliquant en quoi ils sont l'incarnation de certains principes et certaines méthodes qui ont été développées dans la dernière décennie par la communauté du chaos quantique.

5 mars 2018. Au cours de cette première séance, il s'agissait de planter le décor. Le cours s'est donc divisé en deux parties principales. Dans la première, on est revenu sur les arguments de Minakshisundaram-Pleijel de 1949, traitant du noyau de la chaleur, pour expliquer quelques concepts essentiels tels que le théorème spectral, la loi de Weyl, les formules de traces, et les paramétrices.

La deuxième partie a été consacrée à la présentation d'éléments d'analyse microlocale, en motivant leur introduction par l'étude d'équations aux dérivées partielles linéaires. Partant d'observations élémentaires, on a vu d'où proviennent les notions de «symbole principal», de «front d'onde » et de «propagation des singularités». C'était aussi l'occasion de voir apparaître un argument de commutateur positif, ainsi que des équations de transport dans des variétés caractéristiques et des développements BKW.

12 mars 2018. La deuxième séance a elle aussi été divisée en deux parties introductives. D'abord, on a rappelé un certain nombre de concepts et de résultats en géométrie des variétés de courbure négative et de géométrie finie, et la dynamique de leur flot géodésique. C'était l'occasion d'introduire l'objet géométrique principal du cours, les variétés à pointes hyperboliques, et de poser les définitions de la série de Poincaré et de la pression associée à un potentiel.

Ensuite, on a détaillé la structure de la décomposition spectrale du laplacien sur de telles variétés. Après avoir introduit les séries d'Eisenstein, la matrice et le déterminant de diffusion, on a rappelé comment, en courbure constante, apparaissent des séries de Dirichlet, et ce que cela implique sur la distribution des résonances du laplacien.

19 mars 2018. Ce jour, l'objectif était de présenter une paramétrice pour le déterminant de diffusion des variétés à pointes. Celle-ci admet un développement en séries de Dirichlet dans certaines régions du plan complexe. Après avoir donné une ébauche de preuve en courbure constante, il s'est agi de montrer que l'extension à la 
courbure variable repose sur la construction d'une paramétrice BKW pour les séries d'Eisenstein. Celle-ci a ensuite été donnée en plus de détails.

L'objectif suivant était d'expliquer que cela était une incarnation de la méthode de Pression pour construire des paramétrices de fonction de Green. Après en avoir donné une description générale, plusieurs exemples d'applications issus de la littérature ont été passés en revue.

26 mars 2018. Pour ce dernier exposé, il s'agissait d'expliquer comment on peut montrer le prolongement méromorphe de la résolvante du flot géodésique pour des variétés à pointes. On peut ainsi définir un spectre de Pollicott-Ruelle pour ces variétés. D'abord, on a rappelé les techniques de fonctions de fuite et d'espaces anisotropes du cas compact. Ensuite, on a expliqué comment la non-compacité, qui empêche a priori d'utiliser les techniques Fredholm, peut être surmontée en inversant de façon exacte un modèle à l'infini explicite.

\section{Références}

N. Anantharaman, Entropy and the localization of eigenfunctions, Ann. of Math., vol. 168, $\mathrm{n}^{\circ}$ 2, 2008, 435-475, DOI : 10.4007/annals.2008.168.435.

Y. GuEDES BONTHONNEAU, Resonance-free regions for negatively curved manifolds with cusps, Amer. J. Math. , vol. 140, no 3, 2018, 821-877.

Y. COLIN DE VERDIÈRE, Une nouvelle démonstration du prolongement méromorphe des séries d'Eisenstein, C.R. Acad. Sci. Paris, Série I Math., vol. 293, nº 7, 1981, 361-363.

F. FAURE et J. SJÖSTRAND, Upper bound on the density of Ruelle resonances for Anosov flows, Comm. Math. Phys., vol. 308, no 2, 2011, 325-364.

V. GUILlEMIN, Sojourn times and asymptotic properties of the scattering matrix, in: Proceedings of the Oji Seminar on Algebraic Analysis and the RIMS Symposium on Algebraic Analysis (Kyoto Univ., Kyoto, 1976), Res. Inst. Math. Sci., vol. 12, (supplement 1976/77), 1977, 69-88.

Y. Guedes Bonthonneau et T WeIch, Ruelle-Pollicott, Resonances for Manifolds with Hyperbolic Cusps, arXiv e-prints, arXiv:1712.07832, décembre 2017.

S. MinAKSHISUNDARAM et $\AA$. PleIJEL, Some properties of the eigenfunctions of the Laplaceoperator on Riemannian manifolds, Canadian J. Math., vol. 1, 1949, 242-256.

S. NONNENMACHER et M. ZWORSKI, Quantum decay rates in chaotic scattering, Acta Math., vol. 203, n 2, 2009, 149-233.

F. PAUlin, M. PollicotT et B. SCHAPIRA, Equilibrium states in negative curvature, Astérisque, vol. 373, 2015, DOI : 10.24033/ast.975.

A. Selberg, Remarks on the distribution of poles Eisenstein series, in : Collected papers, vol. II, Berlin, Springer, 1989. Avec un avant-propos de K. Chandrasekharan. 